\title{
Erratum to: Predicting children's behaviour during dental treatment under oral sedation
}

\author{
L. Lourenço-Matharu ${ }^{1}$ A. Papineni McIntosh ${ }^{2} \cdot$ J. W. Lo $^{3,4}$
}

Published online: 27 June 2016

(C) European Academy of Paediatric Dentistry 2016

\section{Erratum to: Eur Arch Paediatr Dent (2016) 17:157-163 DOI 10.1007/s40368-015-0205-9}

\section{Page 158}

The third sentence in the first paragraph of the "Study population and design" sub-section should read:

"Children were assessed at a Casualty Clinic or New Patients' Clinic and allocated appropriately for treatment with or without local anaesthesia (LA), with LA and inhalation sedation, LA and oral sedation, LA and intravenous sedation, or general anaesthesia".

\section{Page 161}

The first sentence in the final paragraph of the "Results" section should read:

The online version of the original article can be found under doi:10.1007/s40368-015-0205-9.

L. Lourenço-Matharu

liege.matharu@kcl.ac.uk

1 Unit of Paediatric Dentistry, Dental Institute, King's College Hospital, Bessemer Road, London SE5 9RS, UK

2 Oral and Maxillofacial Surgery Department, Queen Mary's Hospital, King's College Hospitals NHS Foundation Trust, Sidcup, Kent, UK

3 Division of Health and Social Care Research, King's College London, London, UK

4 NIHR Biomedical Research Centre at Guy's and St. Thomas' National Health Service Foundation Trust and King's College London, London, UK
"Chi-squared tests revealed no association between gender and behaviour outcome $(p=0.454)$ or ASA grade and behaviour outcome $(p=0.218)$ ".

\section{Page 161}

The first sentence in the first paragraph of the "Discussion" section should read:

"This study revealed that a high level of child dental fear, as measured by the parental version of CFSS-DS, was a significant predictor of unacceptable behaviour under oral sedation, which agrees with the results of previous studies which have reported that the CFSS-DS scale reliably identifies children who would display dental fear and behavioural problems during dental treatment (Klingberg 1994; Klingberg et al. 1995; Aartman et al. 1998; Wogelius et al. 2003)".

\section{Page 162}

The second sentence in the final paragraph of the "Discussion" section should read:

"Further research in this area could explore midazolam's therapeutic effect on anterograde amnesia. Amnesia alone may not be able to reduce a child's dental anxiety or fear. However, amnesia may be seen as a therapeutic side effect as it does not act to increase or reinforce a child's fear, therefore a child may be more willing to return for further treatment".

\section{Page 162}

The first sentence in the first paragraph of the "Conclusion" section should read:

"Our findings confirmed that a younger age was a predictor of unacceptable behaviour. The study also revealed 
that if a parent perceives their child's dental fear as high, using the CFSS-DS, the child was two times more likely to exhibit unacceptable behaviour under oral sedation than if their parents perceived them to have a low dental fear rating". 